\title{
RESTRINGINDO FLEXIBILIDADE DE PESOS EM DEA UTILIZANDO ANÁLISE DE REGRESSÃO MSEA
}

\author{
Antonio Allen Meireles Alcântara * \\ Programa de Engenharia de Produção - COPPE \\ Universidade Federal do Rio de Janeiro \\ Rio de Janeiro - RJ \\ allen@br-petrobras.com.br
}

\author{
Annibal Parracho Sant'Anna \\ Departamento de Engenharia de Produção \\ Universidade Federal Fluminense \\ Niterói - RJ \\ aparracho@uol.com.br
}

\section{Marcos Pereira Estellita Lins}

Programa de Engenharia de Produção - COPPE

Universidade Federal do Rio de Janeiro

Rio de Janeiro - RJ

estellit@iiis.com.br

* Corresponding author/autor para quem as correspondências devem ser encaminhadas

Recebido em 11/2002, aceito em 06/2003 após 1 revisão

\section{Resumo}

Este artigo apresenta um procedimento baseado no uso de regressão de mínima soma dos valores absolutos dos resíduos (MSAE) para o tratamento dos pesos dos fatores na Análise de Envoltória de Dados (DEA). É desenvolvido um método alternativo para limitar a faixa em que se permite que esses pesos possam variar, no caso de modelos contendo apenas um output.

Palavras-chave: DEA; restrições de peso; MSAE.

\begin{abstract}
In this paper we present a procedure based on the use of Minimum Sum of Absolute Errors (MSAE) regression for the treatment of factor weights in Data Envelopment Analysis (DEA). It develops and presents alternative method to limit the range within which these factor weights are allowed to vary when models using a single output are analyzed.
\end{abstract}

Keywords: DEA; restricted weights; MSAE. 


\section{Introdução}

Neste artigo apresentamos um método para restringir a flexibilidade de pesos dos fatores na utilização de Análise Envoltória de Dados (DEA), metodologia desenvolvida para avaliar eficiência relativa de unidades produtivas através de análises realizadas nos níveis de utilização de seus inputs objetivando a produção de seus outputs. No modelo dos multiplicadores, originalmente proposto por Charnes, Cooper \& Rhodes (1978), as eficiências são resultantes de maximização da razão da soma de outputs ponderados pela soma de inputs ponderados da unidade sob avaliação. Nesta concepção, DEA permite total flexibilidade nos pesos utilizados nas ponderações, de tal forma que cada unidade sob análise, comumente chamada de DMU (Decision Making Unit), alcança sua eficiência máxima não levando em conta qualquer prioridade ou limitação na utilização dos fatores.

Esta flexibilidade permite, por um lado, que cada unidade busque sua eficiência segundo mix de pesos de inputs e outputs próprios, sem necessidade de submeter-se a um único mix para todas as unidades. Por outro lado, esta flexibilidade leva a situações inaceitáveis, devido a duas características dos modelos DEA, segundo Pedraja-Chaparro et al. (1997):

1. As regiões Pareto-ineficientes, onde as DMUs apresentam pesos nulos atribuídos a alguns inputs ou outputs;

2. Os vértices do conjunto de possibilidades de produção, pontos em que se verifica uma infinidade de mix de pesos ótimos (soluções ótimas alternativas do modelo DEA dos multiplicadores).

Freqüentemente em aplicações práticas de DEA, onde não são impostas restrições aos pesos, encontramos soluções impróprias, pois a eficiência de algumas unidades é alcançada quase que exclusivamente devido a um determinado fator. Ocorre, ainda, muitas vezes, as distribuições dos pesos apresentarem-se completamente diferentes para as diferentes unidades.

Este trabalho inicia com uma revisão sumária dos principais estudos sobre restrição de pesos em DEA, propondo a seguir uma discussão sobre novas possibilidades na modelagem DEA, através de um balanceamento mais realista da importância dos fatores utilizando estimação de parâmetros através da técnica MSAE.

O artigo é organizado da seguinte maneira: na Seção 2 são destacados os principais trabalhos sobre restrição de pesos em DEA; na Seção 3 introduzimos a técnica de estimação MSEA apresentando os procedimentos para a obtenção dos estimadores pontuais e por intervalo; na Seção 4 avaliamos a utilização de MSEA aos dados da aplicação, calculamos seus estimadores e a seguir ponderamos os estimadores pelos inputs, terminando com a aplicação da metodologia DEA com e sem restrições aos dados, verificando suas diferenças; finalmente concluímos o artigo na Seção 5 com comentários finais.

\section{Estudos Anteriores}

Diversos trabalhos para impor limites a pesos de fatores têm sido desenvolvidos. Listamos a seguir alguns destes trabalhos que apontam para abordagens que, ao mesmo tempo, procuramos caracterizar.

\section{- Restrições Diretas nos pesos}

Esta abordagem foi desenvolvida por Dyson \& Thanassoulis (1988) e generalizada por Roll, Cook \& Golany (1991). Segundo este método, impõem-se limites numéricos diretamente aos multiplicadores, da forma: 


$$
\begin{gathered}
\mathrm{Q} 2_{\mathrm{i}} \leq \mathrm{V}_{\mathrm{i}} \leq \mathrm{Q} 1_{\mathrm{i}} \text { para os inputs } \\
\mathrm{P} 2_{\mathrm{r}} \leq \mathrm{U}_{\mathrm{r}} \leq \mathrm{P} 1_{\mathrm{r}} \text { para os outputs } \\
\mathrm{B}_{0}=\Sigma \mathrm{V}_{\mathrm{i}} \mathrm{X}_{\mathrm{i} 0}
\end{gathered}
$$

\section{- Região de Segurança (Assurance Region-AR)}

Esta abordagem foi desenvolvida por Thompson, Langemeier, Lee, Lee \& Thrall (1990). As restrições AR são do tipo:

$$
\begin{gathered}
\mathrm{U}_{\mathrm{r}} \leq \alpha_{\mathrm{rs}} \mathrm{U}_{\mathrm{s}}, \forall \mathrm{r} \neq \mathrm{s} \\
\mathrm{V}_{\mathrm{i}} \leq \beta_{\mathrm{ij}} \mathrm{V}_{\mathrm{j}} \forall \mathrm{i} \neq \mathrm{j}
\end{gathered}
$$

onde $\alpha_{\mathrm{rs}}$ e $\beta_{\mathrm{ij}}$ são definidos pelo usuário.

Esta é a chamada Região de Segurança de tipo I (Assurance Region I - AR I)

A Região de Segurança de tipo II (Assurance Region II - AR II), apresentada por Thompson et al. (1990), relaciona os pesos dos inputs e dos outputs.

\section{- Método Cone Ratio}

Esta abordagem foi desenvolvida por Charnes, Cooper, Wei \& Huang (1989). Este método permite uma transformação da base de dados original de modo a que o modelo com restrições possa ser implementado em um software para modelos DEA básicos. Uma vez obtidos os resultados, os dados devem ser transformados para a forma original a fim de serem interpretados.

\section{- Restrição aos inputs e outputs virtuais}

Do ponto de vista do usuário, é, as vezes difícil estabelecer limites aos multiplicadores, levando em conta as unidades de medidas dos inputs e outputs. Uma alternativa mais amigável é estabelecer limites $\phi_{\mathrm{r}}$ e $\psi_{\mathrm{r}}$ ao output virtual $\mathrm{r}$ de uma DMU j, o que foi proposto por Wong \& Beasley (1990). A restrição ao output virtual r é da forma

$$
\phi_{r} \leq \frac{U_{r} y_{r j}}{\sum_{r=1}^{s} U_{r} y_{r j}} \leq \psi_{r},
$$

onde $\sum_{r=1}^{s} U_{r} y_{r j}$ representa o output virtual total da DMU j. Este tipo de restrição, no entanto, acarreta problemas de inviabilidade de difícil solução.

\section{- Restrição contingente dos inputs e outputs virtuais}

Esta abordagem propõe a imposição de limites relativos às participações de cada input (ou output) no input virtual, e foi proposta por Pedraja-Chaparro et al. (1997). Produz restrições da seguinte forma:

$$
\mathrm{c}_{\mathrm{i}} \mathrm{V}_{1} \mathrm{X}_{1 \mathrm{j}} \leq \mathrm{V}_{\mathrm{i}} \mathrm{X}_{\mathrm{ij}} \leq \mathrm{d}_{\mathrm{i}} \mathrm{V}_{1} \mathrm{X}_{1 \mathrm{j}},
$$

para $\mathrm{i}>1$, onde $\mathrm{c}_{\mathrm{i}}$ e $\mathrm{d}_{\mathrm{i}}$ são determinados pelo usuário.

Este tipo de restrição aos pesos é "contingente" porque o padrão dos pesos selecionados depende dos níveis dos inputs e outputs utilizados pela DMU. 
Os métodos apresentados para introduzir as restrições nos pesos podem ser classificados da seguinte forma (Pedraja-Chaparro et al., 1997):

\begin{tabular}{|l|l|l|}
\hline \multirow{2}{*}{ Pesos (multiplicadores) } & \multicolumn{2}{|c}{ Tipo de Restrição } \\
\cline { 2 - 3 } Original & \multicolumn{1}{|c}{ Absoluta } & \multicolumn{1}{c}{ Relativa } \\
\hline & $\begin{array}{l}\text { Dyson \& Thanassoulis (1988) } \\
\text { Roll, Cook \& Golany (1991) } \\
\text { Método Cone Ratio (1989) }\end{array}$ & $\begin{array}{l}\text { Thompson, Langemeier, Lee, } \\
\text { Lee \& Thrall (1990) }\end{array}$ \\
\hline Virtual & Wong \& Beasley (1990) & Pedraja-Chaparro et al. (1997) \\
\hline
\end{tabular}

O critério adotado neste trabalho utiliza o ajustamento preliminar de um modelo de regressão linear y $=\Sigma \mathrm{x}_{\mathrm{i}} \beta_{\mathrm{i}}+\boldsymbol{\varepsilon}$, onde o parâmetro $\beta_{i}$ indica a mudança esperada na variável dependente $\mathrm{y}$ por unidade adicionada à variável $x_{i}$, quando as outras coordenadas do vetor $\mathrm{x}$ de variáveis explicativas permanecem inalteradas. No nosso caso, seguindo esse conceito, os parâmetros $\beta_{i}$ representam as importâncias relativas de cada input na determinação do output y. Depois de estimados estes parâmetros, calculamos intervalos de confiança para as estimativas dos $\beta^{\prime}$ 's. Os limites $\beta_{\min }$ e $\beta_{\max }$ destes intervalos, serão utilizados na construção de proporções que delimitarão os pesos dos inputs na determinação das eficiências utilizando DEA.

Dentro das diversas alternativas de estimação de parâmetros em regressão linear, como por exemplo a popular Mínima Soma dos Erros Quadráticos (MSEQ), a Mínima Soma dos Erros Relativos (MSEA) e também a Mínima Soma dos Erros Absolutos (MSEA), optamos pelo último método, pois se apresenta como a alternativa mais robusta. A busca de alternativas robustas se justifica por ser a presença de outliers uma provável causa dos pesos extremos que queremos evitar. Adotando o algoritmo de minimização da soma dos valores dos resíduos na regressão linear, estaremos sendo coerentes com uma idéia central de DEA, que é a de não descartar outliers, admitindo a existência de informações relevantes neste tipo de observação. Nos últimos anos, tem sido conferida grande atenção à análise de outliers e observações influentes em DEA, formando uma ampla literatura sobre o assunto. Destacamos os tratamentos oferecidos em Pastor, Ruiz \& Sirvent (1999), Wilson (1995), Wilson (1993) e Ruiz \& Sirvent (2001).

Ao contrário de grande parte das abordagens anteriores, a utilização de regressão neste trabalho aparece como um complemento, e não como uma alternativa à metodologia DEA. Nosso objetivo é oferecer uma possibilidade de refinamento para propiciar que seus resultados se apresentem coerentes com a realidade das aplicações.

\section{A Regressão MSEA}

\subsection{Introdução}

O algoritmo da Mínima Soma dos Erros Quadráticos (MSEQ) há muito tempo ocupa posição privilegiada na estimação dos parâmetros de modelos de regressão linear devido, principalmente, à relativa simplicidade da teoria. Tem presença obrigatória em todas as rotinas de computadores para cálculo de estimadores. Entretanto, seus resultados têm de ser questionados quando a distribuição das perturbações não é Normal. A presença de outliers, muitas vezes, revela que o erro aleatório possui uma distribuição complicada pela mistura de alguma parcela discreta ou representante de uma família de distribuições com cauda mais acentuada, como, por exemplo, as de Cauchy \& Laplace (ver Blaterg \& Sargent, 1971). 
Um método para avaliarmos esta distribuição dos erros, apresentado em Montgomery \& Peck (1992), fornece um bom critério para verificarmos se estimadores de MSEQ devem ser utilizados em determinada aplicação. $\mathrm{O}$ método consiste em plotar em ordem crescente os erros, ou seja, $\mathrm{e}_{[1]}<\mathrm{e}_{[2]}<\ldots<\mathrm{e}_{[\mathrm{n}]}$ contra a probabilidade cumulativa $\mathrm{P}_{\mathrm{i}}=[(\mathrm{i}-1 / 2)] / \mathrm{n}$. O gráfico resultante, dependendo de sua forma, indicará presença ou não de outliers que, em caso positivo, sugere a utilização de outra alternativa para os estimadores.

O Método das Mínimas Somas dos Erros Absolutos (MSEA) supera as deficiências encontradas pelo MSEQ na existência de outliers. Este método é menos sensível à existência de dados extremos, podendo ser demonstrado que estimadores de mínima soma dos erros absolutos são de máxima verossimilhança quando os erros seguem uma distribuição de Laplace, conforme Blatberg \& Sargent (1971).

Durante muito tempo, a falta de algoritmos computacionais para min $\Sigma\left|y_{i}-x_{i} \beta\right|$ em modelos de regressão linear múltipla foi uma barreira à utilização do método. Uma contribuição de Charnes, Cooper \& Ferguson (1955) foi formular a regressão MSEA como um problema de programação linear. Nas últimas décadas vários algoritmos eficientes têm sido propostos, ver Barrodale \& Roberts (1973) e Zhang (1993).

Ao trabalharmos regressão linear em conjunto com DEA, onde outliers são importantes nas análises, devemos escolher um método que supere este problema de alta sensibilidade aos dados extremos sem admitir a exclusão de dados. Esta é uma vantagem da regressão de Mínima Soma dos Erros Absolutos (MSEA) sobre outros métodos apresentados em Narula \& Wellington (1985), como alternativas robustas à regressão MSEQ.

\subsection{Estimadores de MSEA}

Considere o modelo de regressão linear múltiplo:

$$
y=\beta_{0}+\beta_{1} x_{I}+\ldots+\beta_{k}+\varepsilon,
$$

onde $y$ é uma variável dependente; $x_{1}, \ldots, x_{k}$ são $k$ variáveis regressoras; $\beta_{0}, \beta_{l}, \ldots \beta_{k}$ são $k+1$ constantes desconhecidas e $\varepsilon$ representa o erro aleatório produzido pelo modelo possuindo função de densidade de probabilidade denotada por $\mathrm{f}(\varepsilon)$.

O estimador $\hat{\beta}$ obtido pelo método da Mínima Soma dos Erros Absolutos (MSEA), para $\beta$, minimiza $\sum_{\mathrm{i}}^{\mathrm{n}}=\left|\mathrm{y}_{\mathrm{i}}-\mathrm{x}_{\mathrm{i}} \beta\right|$ para todos os valores de $\beta$, onde $\mathrm{y}_{\mathrm{i}}$ é o i-ésimo elemento do vetor $\underline{\mathbf{Y}}$ e $\mathrm{x}_{\mathrm{i}}$ é a i-ésima linha da matriz $\mathbf{X}$, sendo $n$ o número de observações. O modelo em (1) pode ser escrito na forma matricial como $\mathbf{Y}=\mathbf{X} \boldsymbol{\beta}+\boldsymbol{\varepsilon}$. O procedimento para encontrarmos o estimador MSEA pode ser formulado através do seguinte PPL:

Minimizar $1 \mathrm{e}^{+}+\mathbf{1}^{\prime} \mathrm{e}^{-}$

sujeito a $\quad \mathrm{X} \boldsymbol{\beta}+\mathrm{e}^{+}-\mathrm{e}^{-}=\mathbf{Y}$

$\mathrm{e}^{+}, \mathrm{e}^{-} \geq \mathbf{0}$

\section{$\beta$ irrestrito em sinal,}

onde o estimador MSEA $\boldsymbol{\beta}$ é um vetor $k \times 1,1$ é um vetor $n \times 1$, sendo

$$
\begin{array}{ll}
\mathbf{e}_{\mathbf{j}}^{+}=\left(\mathbf{y}_{\mathbf{j}}-\mathbf{x}_{\mathrm{j}} \boldsymbol{\beta}\right) & \text { se } \mathbf{y}_{\mathbf{j}}-\mathbf{x}_{\mathbf{j}} \boldsymbol{\beta} \geq \mathbf{0} \\
\mathbf{e}_{\mathrm{j}}^{-}=-\left(\mathbf{y}_{\mathbf{j}}-\mathbf{x}_{\mathbf{j}} \boldsymbol{\beta}\right) & \text { se } \mathbf{y}_{\mathbf{j}}-\mathbf{x}_{\mathbf{j}} \boldsymbol{\beta}<\mathbf{0}
\end{array}
$$




\section{Um exemplo na utilização de MSEA com DEA}

\subsection{Obtenção dos estimadores}

Para facilitar a comparação com outros possíveis métodos de ajustamento do modelo linear, ilustraremos a utilização de estimadores MSEA através da aplicação a dados de um exemplo clássico, aos quais ao critério de mínimos quadrados se aplica satisfatoriamente. A aplicação do algoritmo baseado na minimização da soma da soma dos desvios absolutos a este conjunto de dados ajuda, também, a demonstrar a flexibilidade da metodologia proposta. Os dados, com 25 observações para duas variáveis regressoras, $\mathrm{X}_{1}$ e $\mathrm{X}_{2}$, e uma variável dependente, Y, foram obtidos em Montgomery \& Peck (1992).

Estimamos os coeficientes do modelo de regressão linear pelo critério MSEA, resolvendo o PPL da Seção 3.2. O modelo ajustado é:

$$
\hat{\mathrm{Y}}=3.6621+1.4272 \mathrm{X}_{1}+0.0143 \mathrm{X}_{2}
$$

Utilizando resultados de inferência estatística baseados em Narula (1987), calculamos intervalos de confiança para os estimadores de MSEA da regressão (2).

Para $\beta_{\mathrm{i}}$, ao nível de confiança de $(1-\alpha) 100 \%$ temos o intervalo de confiança:

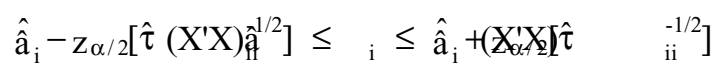

onde $z_{\alpha / 2}$ representa $o(1-\alpha / 2)$ percentil da distribuição da normal padrão sendo $\left.\hat{\tau}=n^{* 1 / 2}\left[e_{[n}{ }^{*}-m+1\right]-e_{[m]}\right] / 4$ um estimador consistente de $\tau$ proposto em McKean \& Schrader (1987), e $\left(\mathrm{X}^{\prime} \mathrm{X}\right)_{\mathrm{ii}}^{-1}$ é o i-ésimo elemento da diagonal da matriz de $\left(\mathrm{X}^{\prime} \mathrm{X}\right)^{-1}$.

Temos ainda $\mathrm{m}=\left[\left(\mathrm{n}^{*}+1\right) / 2-\mathrm{n}^{*}\right]$ com $\mathrm{n}^{*}$ definido como o número de resíduos diferentes de zero e $e_{[1]}, e_{[2]}, e_{[3]}, \ldots, e_{\left[{ }^{*}\right]}$ sendo os resíduos diferentes de zero ordenados em ordem crescente.

No exemplo em estudo, admitindo um intervalo de confiança de $95 \%$, ou seja, $\alpha / 2=0.005$

$$
\begin{aligned}
& \left(X^{\prime} X\right)_{11}^{-1 / 2}=0.05196, \\
& \mathrm{n}^{*}=22, \\
& m=(22+1) / 2-4.69=6.81 \\
& \hat{\tau}=4.69\left(e_{16}-e_{7}\right) / 4=4.69(1.0868+0.6659) / 4=2.055
\end{aligned}
$$

Assim, obtemos o intervalo de confiança para $\beta_{1}$

$$
1.1773 \leq \beta_{1} \leq 1.6771
$$

Calculando de forma análoga para $\hat{a}_{2}$, utilizando: $\left(\mathrm{X}^{\prime} \mathrm{X}\right)_{22}^{-1 / 2}=0.0011$, obtemos

$$
0.0089 \leq \beta_{2} \leq 0.0196
$$




\subsection{Ponderação pelas Escalas dos Inputs}

É importante observar que, simplesmente analisando as estimativas pontuais obtidas na Seção anterior, não podemos concluir muita coisa a respeito da importância de cada input na determinação do output y. Logicamente, os valores dos estimadores de $\beta_{1}$ e $\beta_{2}$ são influenciados pelas escalas de valores dos correspondentes inputs $\mathrm{x}_{1} \mathrm{e}_{2}$.

Devemos propor um procedimento razoável para eliminarmos esta influência, de forma que tenhamos um parâmetro que indique as reais parcelas de contribuição na determinação do output feita por cada input. Uma maneira simples de obtermos este parâmetro é relacionando os estimadores de $\beta_{\mathrm{i}}$ 's com a média dos dados observados da variável $\mathrm{x}_{\mathrm{i}}$, como indicado na fórmula a seguir, na qual $\mathrm{n}$ indica o número de observações e $\mathrm{k}$ o número de coeficientes:

$$
\Psi_{i}=\frac{\left[\hat{\beta}_{i} \cdot \sum_{j=1}^{n} \mathrm{x}_{\mathrm{ij}} / n\right]}{\sum_{l=1}^{k}\left[\hat{\beta}_{l} \cdot \sum_{j=1}^{n} \mathrm{x}_{\mathrm{lj}} / n\right]}
$$

No caso em estudo, aplicando este procedimento, obtemos os pesos $\Psi_{1}=0,6813$ e $\Psi_{2}=0,3187$.

Estas medidas pontuais são transformadas em intervalos $\left(\Psi_{\mathrm{i} \min }, \Psi_{\mathrm{i} \text { Max }}\right)$, aproveitando as amplitudes dos intervalos de confiança (3) e (4).

$\mathrm{O}$ cálculo das amplitudes nos fornece, para $\beta_{1}$, um percentual de $(1.6771-1.4272)$ / $1.4272=0.1751$, ou $17.51 \%$. Da mesma forma, para $\beta_{2}$ teremos uma amplitude de $(0.0196-0.0143) / 0.0143=0.3706$ ou $37.06 \%$

Utilizando estes percentuais de variação, juntamente com $\Psi_{1}$ e $\Psi_{2}$, encontramos os intervalos possíveis para os pesos dos inputs na determinação do output.

$$
\begin{aligned}
& 0.5632 \leq \Psi_{1} \leq 0.8100 \\
& 0.1900 \leq \Psi_{2} \leq 0.4368
\end{aligned}
$$

\subsection{Utilização de Estimadores MSEA para determinar Restrições aos Pesos em DEA}

Utilizando os dados referidos, de Montgomery \& Peck (1992), geramos a Tabela A (ANEXO), com os escores de eficiência, os respectivos conjuntos referência (peer groups) e ainda a contribuição de cada input na obtenção da eficiência. O modelo utilizado foi o de Charnes, Cooper \& Rhodes (1978), com orientação input, indicado na literatura pela sigla CCR-I, sem restrição aos pesos dos fatores como formalizado a seguir.

Seja a $\mathbf{D M U}$ a unidade a ser avaliada. Resolvemos o seguinte problema de programação fracional para obter os valores dos pesos dos inputs $\mathrm{v}_{\mathrm{i}}(\mathrm{i}=1,2)$ e o peso do único output $\mathrm{u}$ como variáveis.

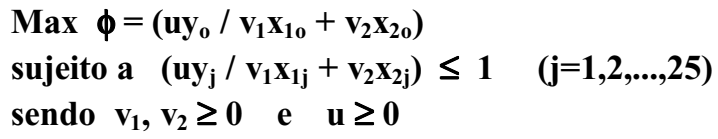

Este é um problema de programação não linear que pode ser convertido em um PPL.

Podemos observar na Tabela $\mathbf{A}$, que todas as observações alcançam suas eficiências baseadas exclusivamente no fator $\mathrm{X}_{1}$, o que, na prática, possivelmente, não se apresenta como um resultado razoável. 
Os pesos representam um valor relativo que proporciona o melhor escore possível para uma determinada unidade, além disso, esse sistema de pesos deve garantir que para todas as unidades, nenhuma alcance um escore de eficiência acima da unidade. Nestas condições, é comum algum fator de menor relevância adquirir um peso relativamente alto, gerando uma unidade eficiente basicamente às custas desse fator, tendo por outro lado atribuído peso nulo a fatores mais relevantes, sendo isso inaceitável do ponto de vista prático.

Utilizando agora o mesmo modelo CCR-I (Equações 6-8) acima, tendo a Equação (8) alterada para

$$
0.5632 \leq \mathrm{v}_{1} \leq 0.8100 \text { e } 0.1900 \leq \mathrm{v}_{2} \leq 0.4368
$$

representando as restrições aos fatores calculadas anteriormente, teremos, aplicando os resultados obtidos por Birkes \& Dodge (1993), os resultados exibidos na Tabela B em anexo.

Como podemos observar na Tabela B, as restrições aos pesos tornaram o resultado da avaliação de desempenho mais balanceada, no sentido de que todos os inputs participam de uma maneira mais aproximada ao ajuste realizado pela regressão linear realizada no início deste trabalho. A contribuição média do input $\mathrm{X}_{1}$ no modelo com restrição utilizando MSAE é de aproximadamente 80,5\% enquanto que inicialmente era 99,7\%. Também se observa, comparando as duas Tabelas, uma maior discriminação entre as observações para o modelo com restrições (Tabela B). De fato, com a aplicação das restrições, a faixa de eficiência tem extremos em 15,5\% e 100,0\%, com apenas uma DMU aparecendo como 100\% eficiente. Esta redução do número de unidades eficientes indica que as restrições impostas cumpriram satisfatoriamente seu papel de evitar que padrões baseados em ponderações extremas elevem os índices de eficiência de algumas unidades.

\section{Comentários Finais}

O ajuste obtido por Mínimos Quadrados por Montgomery \& Peck (1992) é dado pela equação $\mathrm{y}=2.3412+1.6159 \mathrm{x}_{1}+0.0143 \mathrm{x}_{2}$ com $\mathrm{R}^{2}=0.9596$ e os intervalos de confiança produzidos sob as hipóteses clássicas conduzem a restrições para os pesos e resultados finais muito próximos dos que obtivemos com os intervalos de confiança produzidos pelo ajustamento por MSAE. As restrições para os pesos, adotando o critério de mínimos quadrados, seriam de $0.4784 \leq \mathrm{v}_{1} \leq 0.8842$ e $0.0952 \leq \mathrm{v}_{1} \leq 0.5422$. Demonstra-se desta forma a robustez do procedimento proposto para variações na especificação das perturbações. É um procedimento que leva em conta a possível presença de outliers, mas se comporta satisfatoriamente, mesmo quando os dados, ao contrário, se apresentam muito concentrados.

É importante destacar que, mesmo com intervalos de confiança mais largos do que o possivelmente necessário, face à ausência de observações discrepantes no conjunto de dados escolhidos, ainda obtivemos restrições efetivas, no sentido de conduzir a uma envoltória mais realista. Obtemos desta forma um procedimento geral para produção de restrições aos pesos. Este caráter geral, aliado à sua relativa simplicidade, permite que ele seja usado de forma automática, em lugar dos procedimentos ad hoc.

Finalmente, acreditamos que a técnica de restrição de multiplicadores proposta neste artigo, aplicada a somente um output, pode ser generalizada a situações de vários outputs. Uma recomendação para estudos posteriores seria a utilização prévia de correlação canônica, conforme Arnold et al. (1996). 


\section{Referências Bibliográficas}

(1) Arnold, V.; Bardhan, I.; Cooper, W. \& Kumbhakar, S. (1996). New uses of DEA and statistical regressions for efficiency evaluation and estimation. Annals of Operations Research, 66, 255-277.

(2) Barrodale, I. \& Roberts, F.D. (1973). An improved algorithm for discrete $L_{1}$ linear approximation. S.I.AM., J. Numer. Anal., 10, 839-848.

(3) Birkes, D. \& Dodge, Y. (1993). Alternative Methods of Regression. John Wiley and Sons, Inc., New York.

(4) Blaterg, R. \& Sargent, T. (1971). Regression With Non-Gaussian Stable Disturbances: Some Sampling Results. Econometrica, 39(3), 501-510.

(5) Charnes, A.; Cooper, W. \& Ferguson, R. (1955). Optimal estimation of executive compensation by linear programming. Management Science, 1, 138-151.

(6) Charnes, A.; Cooper, W. \& Rhodes, E. (1978). Measuring efficiency of decision making. European Journal of Units Operational Research, 2(6), 429-444.

(7) Charnes, A.; Cooper, W.; Wei, Q.L. \& Huang, Z.M. (1989). Cone Ratio Data Envelopment Analysis and Multiple Objective Linear Programming. International Journal of Management Science, 20(7), 1099-1118.

(8) Dyson, R.G. \& Thanassoulis, E. (1988). Reducing Weight Flexibility in Data Envelopment Analysis. Journal of the Operational Research Society, 39(6), 563-576.

(9) Montgomery, D.C. \& Peck, E.A. (1992). Introduction to Linear Regression Analysis. Wiley \& Sons.

(10) Mckean, J.W. \& Schrader, R.M. (1987). Least absolute errors analysis of variance. In: Statistical Data Analysis Based on the $L_{1}$-Norm and Related Methods [edited by Y. Dodge], Elsevier Science Publishers B, 297-305.

(11) Narula, S. \& Wellington, J. (1985). Interior Analysis for the Minimum Sum of Absolute Errors Regression. Technometrics, 27(2), 181-188.

(12) Narula S. (1987). The Minimum sum of absolute errors regression. Journal of Quality Technology, 19, 37-45.

(13) Pastor, J.T.; Ruiz, J.L. \& Sirvent, I. (1999). A Statistical Test for Detecting Influential Observations in DEA. European Journal of Operational Research, 115(3), 542-554.

(14) Pedraja-Chaparro, R.; Salinas-Jimenes, J. \& Smith, P. (1997). On the Role of Weight Restrictions in DEA. Journal of Productivity Analysis, 8, 215-230.

(15) Roll, Y.; Cook, W. \& Golany, B. (1991). Controlling Factor weights in DEA. IIEE Transactions, 23, 2-9.

(16) Ruiz, J.L. \& Sirvent, I. (2001). Techniques for the assessment of influence in DEA. European Journal of Operational Research, 132, 390-399.

(17) Thompson, R.G.; Langemeier, L.N.; Lee, C.; Lee, E. \& Thrall, R. (1990). The Role of Multiplier Bounds in Efficiency Analysis with Application to Kansas Farming. Journal of Econometrics, 46, 93-108. 
(18) Wilson, P.W. (1993). Detecting Outliers in Deterministic Nonparametric Frontier Models with Multiple Outputs. American Statistical Association, 11(3), 319-323.

(19) Wilson, P.W. (1995). Detecting Influential Observations in Data Envelopment Analysis. The Journal of Productivity Analysis, 6, 27-45.

(20) Wong, Y.H.B. \& Beasley, J.E. (1990). Restricting Weight Flexibility in DEA. Journal of the Operational Research Society, 41, 829-835.

(21) Zhang, Y. (1993). Primal-Dual interior point approach for computing $1_{1}$ solutions and $1_{\mu}$ solutions of overdetermined systems. Journal of Optimization Theory and Applications, 77, 323-341.

\section{ANEXO}

Tabela A

\begin{tabular}{|c|c|c|c|c|}
\hline \multicolumn{5}{|c|}{$\begin{array}{c}\text { Eficiência das unidades, contribuição dos inputs e referências } \\
\text { Sem Restrição de Multiplicadores - Modelo CCR-I }\end{array}$} \\
\hline Observação & Eficiência \% & Referência & Contribuição X $\mathbf{1}_{1}$ & Contribuição $\mathbf{X}_{\mathbf{2}}$ \\
\hline 19 & 100,00 & - & $0,24596(97,11 \%)$ & $0,00728(2,87 \%)$ \\
\hline 7 & 100,00 & - & $0,44500(99,77 \%)$ & $0,00100(0,22 \%)$ \\
\hline 4 & 100,00 & - & $0,15703(97,12 \%)$ & $0,00464(2,87 \%)$ \\
\hline 10 & 100,00 & - & $0,17442(99,88 \%)$ & $0,00021(0,12 \%)$ \\
\hline 3 & 94,02 & 7 e 10 & $0,29307(99,88 \%)$ & $0,00035(0,12 \%)$ \\
\hline 2 & 93,88 & 10 & $0,30612(99,88 \%)$ & $0,00037(0,12 \%)$ \\
\hline 13 & 83,54 & 10 & $0,23207(99,88 \%)$ & $0,00028(0,12 \%)$ \\
\hline 14 & 80,28 & 7 & $0,15244(99,88 \%)$ & $0,00121(0,12 \%)$ \\
\hline 18 & 74,55 & 19 & $0,09168(97,12 \%)$ & $0,00271(2,87 \%)$ \\
\hline 16 & 70,68 & 7 e 10 & $0,09140(99,88 \%)$ & $0,00011(0,12 \%)$ \\
\hline 25 & 70,32 & 4 e 7 & $0,23057(99,77 \%)$ & $0,00052(0,22 \%)$ \\
\hline 15 & 67,37 & 4 & $0,09993(99,77 \%)$ & $0,00022(0,22 \%)$ \\
\hline 8 & 67,03 & 7 & $0,13383(99,77 \%)$ & $0,00030(0,22 \%)$ \\
\hline 9 & 66,88 & 4 e 7 & $0,30050(99,77 \%)$ & $0,00006(0,22 \%)$ \\
\hline 17 & 66,86 & 7 & $0,15505(99,77 \%)$ & $0,00035(0,22 \%)$ \\
\hline 6 & 65,71 & 7 & $0,12917(99,77 \%)$ & $0,00029(0,22 \%)$ \\
\hline 11 & 64,57 & 7 & $0,05699(99,77 \%)$ & $0,00013(0,22 \%)$ \\
\hline 5 & 60,95 & 7 & $0,15780(99,77 \%)$ & $0,00035(0,22 \%)$ \\
\hline 24 & 60,30 & 7 e 10 & $0,11403(99,88 \%)$ & $0,00013(0,12 \%)$ \\
\hline 1 & 57,93 & 10 & $0,13023(99,88 \%)$ & $0,00016(0,12 \%)$ \\
\hline 12 & 56,27 & 7 & $0,09539(99,77 \%)$ & $0,00021(0,22 \%)$ \\
\hline 21 & 54,16 & 19 & $0,07070(97,12 \%)$ & $0,00209(2,87 \%)$ \\
\hline 22 & 52,83 & 7 & $0,03594(99,77 \%)$ & $0,00008(0,22 \%)$ \\
\hline 20 & 52,64 & 7 & $0,05339(99,77 \%)$ & $0,00012(0,22 \%)$ \\
\hline 23 & 52,61 & 7 & $0,09989(99,77 \%)$ & $0,00022(0,22 \%)$ \\
\hline
\end{tabular}


Tabela B

\begin{tabular}{|c|c|c|c|c|}
\hline \multicolumn{5}{|c|}{$\begin{array}{c}\text { Eficiência das unidades, contribuição dos inputs e referências } \\
\text { Com Restrição de Multiplicadores - Modelo CCR-I }\end{array}$} \\
\hline Observação & Eficiência \% & Referência & Contribuição $\mathbf{X}_{1}$ & Contribuição X 2 \\
\hline 19 & 100,0 & - & $0,08658(80,81 \%)$ & $0,02056(19,19 \%)$ \\
\hline 4 & 78,60 & 19 & $0,04348(80,80 \%)$ & $0,01033(20,20 \%)$ \\
\hline 18 & 60,20 & 19 & $0,02608(79,58 \%)$ & $0,00619(20,42 \%)$ \\
\hline 21 & 50,30 & 19 & $0,02312(80,81 \%)$ & $0,00549(19,19 \%)$ \\
\hline 12 & 41,80 & 19 & $0,01638(80,81 \%)$ & $0,00389(19,19 \%)$ \\
\hline 5 & 40,20 & 19 & $0,02402(80,79 \%)$ & $0,00571(19,21 \%)$ \\
\hline 8 & 38,10 & 19 & $0,01758(80,79 \%)$ & $0,00418(19,21 \%)$ \\
\hline 17 & 34,90 & 19 & $0,01869(80,80 \%)$ & $0,00444(19,20 \%)$ \\
\hline 7 & 34,60 & 19 & $0,03556(80,82 \%)$ & $0,00844(19,18 \%)$ \\
\hline 25 & 33,30 & 19 & $0,02524(80,82 \%)$ & $0,00599(19,18 \%)$ \\
\hline 22 & 29,10 & 19 & $0,00458(80,78 \%)$ & $0,00109(19,22 \%)$ \\
\hline 11 & 27,30 & 19 & $0,00557(80,84 \%)$ & $0,00132(19,16 \%)$ \\
\hline 6 & 25,8 & 19 & $0,01171(80,81 \%)$ & $0,00278(19,19 \%)$ \\
\hline 9 & 25,6 & 19 & $0,00265(80,79 \%)$ & $0,00063(19,21 \%)$ \\
\hline 13 & 25,40 & 19 & $0,01549(80,80 \%)$ & $0,00368(19,20 \%)$ \\
\hline 15 & 25,30 & 19 & $0,00867(80,80 \%)$ & $0,00206(19,20 \%)$ \\
\hline 2 & 25,30 & 19 & $0,01810(81,06 \%)$ & $0,00423(18,94 \%)$ \\
\hline 20 & 21,30 & 19 & $0,00500(80,77 \%)$ & $0,00119(19,23 \%)$ \\
\hline 14 & 20,70 & 19 & $0,00864(80,82 \%)$ & $0,00205(19,18 \%)$ \\
\hline 23 & 19,70 & 19 & $0,00863(80,80 \%)$ & $0,00205(19,20 \%)$ \\
\hline 16 & 18,10 & 19 & $0,00515(80,85 \%)$ & $0,00122(19,15 \%)$ \\
\hline 10 & 17,60 & 19 & $0,00672(80,87 \%)$ & $0,00159(19,13 \%)$ \\
\hline 3 & 17,50 & 19 & $0,01194(80,78 \%)$ & $0,00284(19,22 \%)$ \\
\hline 24 & 15,20 & 19 & $0,00629(80,85 \%)$ & $0,00149(19,15 \%)$ \\
\hline 1 & 14,50 & 19 & $0,00714(80,86 \%)$ & $0,00169(19,14 \%)$ \\
\hline
\end{tabular}

